\title{
Utility of Plasma Neurofilament Light in the 1Florida Alzheimer's Disease Research Center (ADRC)
}

Warren Barker ${ }^{\mathrm{a}}$, Carlos Quinonez ${ }^{\mathrm{a}}$, Maria T. Greig ${ }^{\mathrm{a}}$, Raquel Behar ${ }^{\mathrm{a}}$, Cesar Chirinos ${ }^{\mathrm{a}}$, Rosemarie A. Rodriguez ${ }^{\mathrm{a}}$, Monica Rosselli ${ }^{\mathrm{b}}$, Miriam J. Rodriguez ${ }^{\mathrm{c}}$, Rosie Curiel Cid ${ }^{\mathrm{d}}$, Tatjana Rundek ${ }^{\mathrm{e}}$, Karen McFarland ${ }^{\mathrm{f}}$, Kevin Hanson ${ }^{\mathrm{f}}$, Glenn Smith ${ }^{\mathrm{f}}$, Steven DeKosky ${ }^{\mathrm{f}}$, David Vaillancourt ${ }^{\mathrm{f}}$, Malek Adjouadi ${ }^{\mathrm{g}}$, Michael Marsiske ${ }^{\mathrm{f}}$, Nilufer Ertekin-Taner ${ }^{\mathrm{h}, \mathrm{i}}$, Todd Golde ${ }^{\mathrm{f}}$, David A. Loewenstein ${ }^{\mathrm{d}}$ and Ranjan Duara ${ }^{\mathrm{a}, *}$

${ }^{a}$ Wien Center for Alzheimer's Disease and Memory Disorder, Mount Sinai Medical Center, Miami Beach, FL, USA

${ }^{\mathrm{b}}$ Florida Atlantic University, Department of Psychology, Charles E. Schmidt College of Science, Davie, FL, USA ${ }^{\mathrm{c} A l b i z u}$ University, Miami, FL, USA

${ }^{\mathrm{d}}$ Department of Psychiatry and Behavioral Sciences and Neurology, Miller School of Medicine, University of Miami, FL, USA

${ }^{\mathrm{e}}$ Department of Neurology, Miller School of Medicine, University of Miami, Miami, FL, USA

${ }^{\mathrm{f}}$ Florida ADRC, University of Florida, Gainesville, FL, USA

${ }^{\mathrm{g}}$ College of Engineering and Computing, Florida International University, Miami, Florida, USA

${ }^{\mathrm{h}}$ Mayo Clinic Florida, Department of Neuroscience, Jacksonville, FL, USA

${ }^{\mathrm{i}}$ Mayo Clinic Florida, Department of Neurology, Jacksonville, FL, USA

Accepted 12 October 2020

\begin{abstract}
.
Background: Plasma NfL (pNfL) levels are elevated in many neurological disorders. However, the utility of pNfL in a clinical setting has not been established.

Objective: In a cohort of diverse older participants, we examined: 1) the association of pNfL to age, sex, Hispanic ethnicity, diagnosis, and structural and amyloid imaging biomarkers; and 2) its association to baseline and longitudinal cognitive and functional performance.

Methods: 309 subjects were classified at baseline as cognitively normal $(\mathrm{CN})$ or with cognitive impairment. Most subjects had structural MRI and amyloid PET scans. The most frequent etiological diagnosis was Alzheimer's disease (AD), but other neurological and neuropsychiatric disorders were also represented. We assessed the relationship of pNfL to cognitive and functional status, primary etiology, imaging biomarkers, and to cognitive and functional decline.

Results: pNfL increased with age, degree of hippocampal atrophy, and amyloid load, and was higher in females among CN subjects, but was not associated with Hispanic ethnicity. Compared to $\mathrm{CN}$ subjects, pNfL was elevated among those with $\mathrm{AD}$ or FTLD, but not those with neuropsychiatric or other disorders. Hippocampal atrophy, amyloid positivity and higher pNfL levels each added unique variance in predicting greater functional impairment on the CDR-SB at baseline. Higher baseline pNfL levels also predicted greater cognitive and functional decline after accounting for hippocampal atrophy and memory scores at baseline.
\end{abstract}

\footnotetext{
${ }^{*}$ Correspondence to: Ranjan Duara, MD, Wien Center for Alzheimer's Disease and Memory Disorder, Mount Sinai Medical Center, 4300 Alton Road, Miami Beach, FL 33140, USA. Tel.: +1 305674 2543; E-mail: ranjan.duara@msmc.com.
} 
Conclusion: pNfL may have a complementary and supportive role to brain imaging and cognitive testing in a memory disorder evaluation, although its diagnostic sensitivity and specificity as a stand-alone measure is modest. In the absence of expensive neuroimaging tests, pNfL could be used for differentiating neurodegenerative disease from neuropsychiatric disorders.

Keywords: Alzheimer's disease, amyloid, diagnosis, hippocampal atrophy, magnetic resonance imaging, plasma neurofilament light, positron emission tomography

\section{INTRODUCTION}

Mixed pathologies are the most common cause of cognitive impairment, although $\mathrm{AD}$ is the most frequent pathology occurring in $65 \%$ of cases. On neuropathological examination, AD occurs in isolation only $9 \%$ of cases [1]. The NIA-AA Research Framework classification, summarized as ATN (Amyloid, Tau, Neurodegeneration), is independent of the clinical syndrome, and provides a template for research studies to link the clinical syndrome to biomarkers of AD pathology and neurodegeneration. To that end, molecular biomarkers in both cerebrospinal fluid (CSF) and blood have been developed to measure the pathological and neurodegenerative changes in the brain associated with $\mathrm{AD}$. These biomarkers in CSF and plasma include $A \beta_{42}$ and $\mathrm{A} \beta_{40}$ associated with the deposition of amyloid- $\beta$ plaques, and total tau (T-tau) and phosphorylated tau (P-tau), associated with neurofibrillary tangles.

Neurofilament light (NfL) is a protein that can be measured in the CSF (cNfL) and blood (pNfL). It is a marker for subcortical large-caliber axonal degeneration [2]. CSF NfL and pNfL concentrations correlate with each other and are associated with cognitive decline and increased brain atrophy, including in the hippocampus [3-6]. Plasma NfL and cNfL concentrations are elevated in many neurological disorders, including AD, frontotemporal lobe degeneration (FTLD), vascular dementia, and dementia with Lewy bodies, as compared to cognitively normal (CN) older subjects [7]. Among CN subjects and in most neurological conditions, cNfL has been shown to increase with age [7]. Compared to women, men have been shown to have elevated $\mathrm{cNfL}$ among $\mathrm{CN}$ older subjects and those with AD [7].

While pNfL has been shown to have potential use as a clinical tool, several gaps in current knowledge exist. For example, it is unknown if elevated pNfL levels distinguish between those with evidence of neurodegeneration and those with cognitive impairment resulting from depression, anxiety and other psychiatric conditions. It is also not clear whether factors such as age and Hispanic ethnicity have a bearing on the diagnostic utility of pNfL levels. The diagnostic utility of pNfL levels, relative to cognitive test performance and other biomarkers such as hippocampal atrophy and amyloid load in the brain is not known. Finally, there is insufficient research to determine whether pNfL levels provide complementary information, in the prediction of disease progression, to established predictors such as imaging measures and memory scores.

In the current study, we evaluated the utility of pNfL levels in the 1Florida Alzheimer's Disease Research Center (1Florida ADRC), a longitudinal study of aging sponsored by NIA as one of the 32 Alzheimer's Disease Research Centers. This cohort is bi-ethnic, with nearly equivalent numbers of Hispanic and non-Hispanic participants, including those who are cognitively normal or have various stages of mild cognitive impairment (MCI). In contrast to the Alzheimer's Disease Neuroimaging Initiative (ADNI), there were no inclusion criteria based on functional or memory scores, and few exclusion criteria. This resulted in a cohort having a continuous spectrum of cognitive levels, including those who are $\mathrm{CN}$, the entire spectrum of amnestic and nonamnestic MCI and those in an intermediate stage of impairment between $\mathrm{CN}$ and MCI. This intermediate stage of impairment is categorized as pre-MCI by some $[8,9]$ and "cognitively impaired/not MCI" by others [10]. In addition, this cohort is diverse with respect to etiology, and includes many non-AD diagnoses such as FTLD, Lewy body disease, vascular cognitive impairment, and neuropsychiatric disorders. About half of the participants in this study were recruited from a memory disorder clinic, making the results more generalizable to a clinic setting. The primary goal of the current study was to determine the utility of pNfL as a clinical tool in the evaluation of $\mathrm{AD}$ and related disorders, as well as in predicting the rate of cognitive and functional decline among subjects with $\mathrm{AD}$. 


\section{METHODS}

\section{Participants}

Participants were enrolled in the 1Florida ADRC. The focus of the 1FloridaADRC is the study of older subjects with normal cognition, MCI, and mild dementia, and those in the gap between $\mathrm{CN}$ and MCI. This cohort is unique due to its ethnic and cultural diversity, as over 50\% identify as Hispanic and report Spanish as their primary language. In addition, subjects were assessed using novel neuropsychological test measures, and most had structural brain MRI, amyloid PET, and apolipoprotein E (APOE) genotyping. About half of this cohort has been recruited from a large outpatient memory disorder clinic and the remainder from a free memory screening program and from outreach efforts in the community.

Participants in this study completed an extensive medical, neurological, psychiatric, and neuropsychological evaluation, including all elements required by the National Alzheimer's Coordinating Center (NACC) Uniform Data Set, version 3.0 [10]. The Clinical Dementia Rating Scale (CDR) [11] was administered by an experienced geriatric psychiatrist (MTG), who was blinded to the neuropsychological test results. The CDR Sum of Boxes (CDR-SB) score was used as a global cognitive/functional measure, since it has been shown to accurately stage the severity of $\mathrm{AD}$ in the spectrum from MCI to severe dementia [12]. Ethnicity of the participants was determined using self-report [13]. For the 309 participants included in this study, blood was drawn at baseline for DNA extraction for genetic studies, including $A P O E$ genotyping, and plasma biomarker studies. Two hundred and forty-four (244) participants had a 1st follow-up visit, and 164 a 2nd follow-up visit. All participants had a study partner who served as a collateral informant for the CDR. This study was approved by the Mount Sinai Medical Center IRB and was conducted in accordance with the declaration of Helsinki. All participants and their study partners provided informed consent

A neuropsychological battery was administered in the participant's preferred language (English or Spanish) by a psychometrician who was blinded to the clinical evaluation and the CDR score. The neuropsychological protocol included items from either Version 2 or Version 3 of the Alzheimer Disease Centers' Neuropsychological Test Battery in the Uniform Data Set (UDS), utilizing the Spanish version [10] as applicable. Additional tests were administered including the Hopkins Verbal Learning Test-Revised (HVLT-R) [14] and the Mini-Mental State Examination (MMSE) [15]. The total recall score for HVLT-R was used in this study.

\section{Diagnostic procedures}

Cognitive diagnoses followed the NACC D1 classification protocol, which specifies the diagnosis of: Cognitively Normal (CN), cognitively impaired, not MCI (CInMCI); amnestic and non-amnestic Mild Cognitive Impairment (MCI), and Dementia (DEM) [10]. Etiological diagnoses for subjects with cognitive impairment were operationalized according to NACC D1 guidelines and consolidated into categories as shown in Table 1): cognitively normal $(\mathrm{CN})$; Alzheimer [16]; neuropsychiatric (primarily anxiety and depression); vascular brain injury (VBI); FTLD; and Lewy body dementia (LBD). The remaining diagnoses were consolidated into "Dx-Other", which included less common causes of cognitive deficits, such as sleep disorder, amyloid angiopathy, chemobrain, and ruptured aneurysm.

\section{MR imaging}

MRI scans were performed using a Siemens Skyra 3T MRI scanner at Mount Sinai Medical Center, Miami Beach. MRI scans were evaluated by visual inspection for assessment of medial temporal atrophy and classified as positive or negative for hippocampal atrophy (Hp+, Hp-) [17]. In addition, FreeSurfer Version 6.0 software was used to obtain parcellation of regional brain volumes and cortical thickness at $1.0 \mathrm{~mm}$ isotropic resolution (http://surfer.nmr.mgh. harvard.edu) and for co-registration with amyloid PET scans [18]. The ratio of hippocampal volume to intracranial volume was used as a continuous measure. Cerebrovascular disease on imaging was assessed using the NACC D1 binary categories for presence or absence of large vessel infarcts, lacunar infarcts, moderate white matter hyperintensities, and extensive white matter hyperintensities.

\section{Amyloid PET imaging}

Amyloid PET scans were performed using the tracer $\left[{ }^{18} \mathrm{~F}\right]$ florbetaben for approximately $85 \%$ of the scans and $\left[{ }^{18} \mathrm{~F}\right]$ Florbetapir for the remainder. The methodology for PET scanning has been previously described [19]. All amyloid PET scans were read initially by an independent, trained radiologist, 
Table 1

Demographics and Clinical Characteristics

\begin{tabular}{|c|c|c|c|c|c|c|c|c|}
\hline & $\mathrm{CN}$ & Alzheimer & $\begin{array}{c}\text { Neuro- } \\
\text { psychiatric* }\end{array}$ & VBI & FTLD & LBD & Dx-Other** & $\begin{array}{l}\text { F or Chi- } \\
\text { Square }(p)\end{array}$ \\
\hline$n($ total $=309)$ & 51 & 156 & 47 & 12 & 6 & 5 & 32 & \\
\hline Age (y) & $70.8^{\mathrm{a}} \pm 5.9$ & $74.8^{\mathrm{b}} \pm 8.2$ & $70.5^{\mathrm{a}} \pm 6.7$ & $74.9^{\mathrm{a}} \pm 4.8$ & $71.6^{\mathrm{a}} \pm 4.5$ & $70.7^{\mathrm{a}} \pm 12.9$ & $69.9^{\mathrm{a}} \pm 6.9$ & $4.6(<0.0001)$ \\
\hline Education (y) & $16.0 \pm 2.8$ & $14.8 \pm 3.3$ & $14.4 \pm 3.8$ & $15.6 \pm 2.5$ & $14.5 \pm 4.0$ & $16.6 \pm 2.2$ & $14.6 \pm 3.8$ & $1.43(0.20)$ \\
\hline Sex (Female/Male) & $40 / 11$ & $88 / 68$ & $36 / 11$ & $4 / 8$ & $2 / 4$ & $0 / 5$ & $21 / 11$ & $24.8(0.0004)$ \\
\hline Race (white/black/other) & $41 / 2 / 8$ & $147 / 5 / 4$ & $46 / 0 / 1$ & $12 / 0 / 0$ & $6 / 0 / 0$ & $5 / 0 / 0$ & $29 / 3 / 0$ & $5.9(0.44)$ \\
\hline Hispanic Ethnicity (\%) & $52.9 \%$ & $60.3 \%$ & $66.0 \%$ & $83.3 \%$ & $50.0 \%$ & $20 \%$ & $56.2 \%$ & $7.2(0.20)$ \\
\hline \multicolumn{9}{|l|}{ Follow-up Visits (number) } \\
\hline Year $2($ total $=244)$ & 46 & 118 & 37 & 11 & 5 & 1 & 26 & \\
\hline Year $3($ total $=164)$ & 37 & 81 & 20 & 8 & 2 & 1 & 15 & - \\
\hline $\begin{array}{l}\text { CDR-SB at Baseline } \\
\text { CDR-SB (change) }\end{array}$ & $0.1^{\mathrm{a}} \pm 0.3$ & $2.9^{b} \pm 2.6$ & $0.8^{\mathrm{a}} \pm 0.8$ & $1.9^{\mathrm{a}} \pm 1.8$ & $3.3^{\mathrm{b}} \pm 2.6$ & $3.2^{\mathrm{b}} \pm 1.0$ & $1.5^{\mathrm{b}} \pm 2.6$ & $12.5(<0.0001)$ \\
\hline Year 1 - Year 2 & $-0.04^{\mathrm{a}} \pm 0.3$ & $-1.1^{\mathrm{b}} \pm 2.1$ & $-0.1^{\mathrm{a}} \pm 0.6$ & $0.1^{\mathrm{a}} \pm 0.4$ & $-3.2^{\mathrm{b}} \pm 2.3$ & & $-0.4^{\mathrm{a}} \pm 0.9$ & $6.46(<0.0001)$ \\
\hline $\begin{array}{l}\text { Year } 2 \text { - Year } 3 \text { (only shown } \\
\text { for } n \geq 5 \text { ) }\end{array}$ & $0.0^{\mathrm{a}} \pm 0.2$ & $-1.2^{\mathrm{b}} \pm 2.2$ & $0.2^{\mathrm{a}} \pm 0.7$ & & & - & $0.0^{\mathrm{a}} \pm 0.6$ & $5.75(<0.0001)$ \\
\hline MMSE Score & $29.4^{\mathrm{a}} \pm 0.8$ & $25.3^{\mathrm{b}} \pm 4.3$ & $28.1^{\mathrm{a}} \pm 2.1$ & $28.5^{\mathrm{a}} \pm 1.7$ & $26.2^{\mathrm{a}} \pm 3.9$ & $27.8^{\mathrm{a}} \pm 2.2$ & $27.5^{\mathrm{a}} \pm 3.2$ & $5.8(0.02)$ \\
\hline HVLT-R Total & $26.0^{\mathrm{a}} \pm 4.6$ & $17.03^{\mathrm{b}} \pm 6.0$ & $21.8^{\mathrm{b}} \pm 5.0$ & $21.3^{\mathrm{a}} \pm 5.8$ & $11.2^{\mathrm{b}} \pm 9.4$ & $18.2^{\mathrm{b}} \pm 4.6$ & $20.6^{\mathrm{b}} \pm 6.2$ & $17.5(<0.0001)$ \\
\hline
\end{tabular}

${ }^{*}$ Anxiety $(n=16)$, Apathy $(n=1)$, Bipolar $(n=1)$, Depression $(n=27)$, Lack of Motivation $(n=1)$, PseudoDementia $(n=1)$; ${ }^{* *}$ Other includes: Amyloid Angiopathy $(n=2)$, Attention Deficit Disorder $(n=1)$, Chemobrain $(n=1)$, Cognitive Impairment NOS $(\mathrm{n}=1)$, Essential tremor $(n=1)$, Herpes Simplex Encephalitis $(n=1)$, Hydrocephalus $(n=1)$, mild behavioral impairment $(n=1)$, Medication $(n=1)$, Multifactor Etiology $(n=1)$, Neurodegenerative Disease $(n=5)$, Personality Disorder $(n=1)$, Ruptured MCA Aneurysm $(n=1)$, Schizophrenia $(n=1)$, Sleep Disorder $(n=5)$, Space Occupying Lesion $(n=2)$, Subjective Memory Disorder $(n=1)$, Unknown 5. CN, cognitively normal; VBI, vascular brain injury; FTLD, Frontotemporal lobar degeneration; LBD, Lewy Body Disease; MMSE, Mini-mental State Examination score; CDR-SB, Clinical Dementia Rating Scale Sum of Boxes; HVLT-R, Hopkins Verbal Learning Test-Revised. F values are adjusted for age, where appropriate. Means with different alphabetic superscripts are statistically significant at $p=0.05$ from $\mathrm{CN}$ at $p=0.05$ by Dunnett's post-hoc procedure.

who was not otherwise involved in the study, and a trained and experienced reader (RD), both of whom were blinded to the cognitive and clinical diagnosis, using a methodology similar to that described by Sabri et al. [20]. Images were displayed using a reader-adjustable gray scale to provide optimal discrimination of the cerebellar gray matter from white matter. A final dichotomous classification of elevated amyloid ( $A+$ versus A-) was rendered. Duara et al. [19] found high agreement between raters in reading these scans.

Quantitative assessment of PET scans was performed using a composite standard uptake value ratio (SUVR) calculated by the ratio of the mean tracer uptake from 6 cortical regions (frontal, temporal, parietal, precuneus, anterior and posterior cingulate cortex regions, each region summed from left and right hemispheres) to the whole cerebellum. A centiloid score for each participant was calculated for Florbetaben [21] and Florbetapir [22] PET scans.

\section{APOE genotyping}

All samples for $A P O E$ genotyping were performed in Dr. Nilüfer Ertekin-Taner's laboratory (Mayo Clinic, Jacksonville, FL, USA); the APOE $\varepsilon 2, \varepsilon 3$, and $\varepsilon 4$ alleles used predesigned TaqMan SNP Genotyping
Assays for SNPs rs7412 and rs429358 (Thermo Fisher Scientific, MA, USA) on the QuantStudio 7 Flex Real-Time PCR system (Applied Biosystems, CA, USA) following the manufacturer's protocol.

\section{Plasma biomarker analysis (using Quanterix single molecule array - SIMOA technology)}

Blood samples were drawn at the baseline visit and centrifuged to obtain plasma. Aliquots were stored at $-80^{\circ} \mathrm{C}$ until the samples were shipped to Quanterix Corporation (113 Hartwell Avenue, Lexington, MA, USA, E-mail: techsupport@ quanterix.com.) for analysis of pNfL utilizing the Simoa NF-light kit on the Quanterix Simoa HD-1 Analyzer. Concentrations of $\mathrm{pNfL}$ were measured, in units of $\mathrm{pg} / \mathrm{ml}$, in duplicate from each sample, that were blinded to demographic and clinical data. All samples tested were within the assay dynamic range with an average coefficient of variation of $3.9 \%$ (range from 0 to $15 \%$ for measurement.

\section{Data analysis}

All analyses were conducted using SAS version 9.4 (SAS Institute Inc., Cary, NC, USA). Plasma NfL had a skewed distribution (based on visual inspection 
of histograms and Kolmogorov-Smirnov statistic in the entire cohort and in the two largest subgroups, $\mathrm{CN}$ and $\mathrm{AD}$ ), so a natural $\log$ transformation was applied for analyses that required normal distribution or normal residuals. Comparisons of means between diagnostic groups, for demographic and clinical variables, were performed using general linear models (GLM). Post-hoc tests were conducted using Dunnett procedure, with the $\mathrm{CN}$ subjects as the post-hoc control group. Differences between groups for categorical variables were evaluated using chi-square tests; post-hoc comparisons were conducted with chisquare tests (or Fisher's exact for cell sizes less than 5) using the $\mathrm{CN}$ subjects as the control group and Bonferroni correction.

The relationship between demographic factors (age, sex, and Hispanic ethnicity) and pNfL was assessed with Pearson correlations or GLM. These relationships were assessed separately in larger etiological subgroups (i.e., $\mathrm{CN}$ and $\mathrm{AD}$ ). The relationship of pNfL to binary and continuous measures of hippocampal atrophy and amyloid load was assessed using Pearson correlations.

To determine differences in pNfL between $\mathrm{CN}$ subjects and those in the 6 diagnostic groups, we performed a GLM, using age as a covariate, and etiological diagnosis as independent variable. The Dunnett post-hoc procedure was used to compare the $\mathrm{CN}$ group to all other groups. Similar analyses were done with binary and continuous variables for hippocampal atrophy and elevated brain amyloid, using GLM or Chi-Square analyses, as a way to compare their utility with pNfL for making an etiological diagnosis.

The association of pNfL to cognitive-functional status was assessed with a GLM using CDR-SB as a dependent measure, and $\mathrm{pNfl}$, presence of amyloid, hippocampal atrophy, and $A P O E$ genotype as independent predictor variables. A second GLM was performed using binary and continuous measures for hippocampal atrophy and amyloid.

Diagnostic accuracy of pNfL for distinguishing $\mathrm{CN}$ from $\mathrm{AD}$ (and other etiologies with elevated pNfL) was assessed using logistic regression after controlling for age and sex. Area under the receiver operating characteristic (AUROC) curve was obtained and Youden's criteria was used to determine the optimal sensitivity and specificity and cut-point.

The utility of pNfL in predicting cognitive-functional decline among subjects with cognitive impairment at baseline was assessed using CDR-SB as a repeated measure in a GLM. CDR-SB has been shown to be a good outcome measure for cognitive decline in longitudinal studies [12]. Plasma NfL, presence of amyloid, hippocampal atrophy, score on the HVLT-R, Immediate, and the number of APOE4 alleles $(0,1,2)$ served as independent predictor variables, with age as a covariate. To assess the ability of pNfL to predict clinical progression, logistic regression analyses were performed to determine how $\mathrm{pNfL}$ and other biomarkers would predict a decline of two or more points on the CDR-SB over 2 years among subjects with cognitive impairment at baseline. A decline of 2 points was based on the typical decline in AD subjects with MCI or mild dementia [23]. All tests were 2-sided. Statistical significance was set at $p<0.05$.

\section{RESULTS}

\section{Demographics and clinical characteristics by disease etiology}

Age

Compared with the $\mathrm{CN}$ group, only subjects in the AD group were older (Table 1). There was a difference in the distribution of sex between the 7 diagnostic groups. The percentage of females in the $\mathrm{CN}$ subjects $(78.4 \%)$ was greater than in those with $\operatorname{AD}(57 \%)$, VBI (33\%), or LBD (0\%). There were no differences between the groups in education, race, or the percentage of subjects reporting Hispanic ethnicity. The CDR-SB score was higher in the AD, FTLD, Dx-Other, and LBD groups than in the CN group. Subjects in the AD group had a lower MMSE score than those in the CN group. On the HVLT-R test, the $\mathrm{CN}$ subjects scored significantly better than subjects in the other groups, except for the VBI group.

\section{Association of pNfL and imaging biomarkers with age, sex, and Hispanic ethnicity}

There was a positive association between $\mathrm{pNfL}$ and age among the $\mathrm{CN}$ and $\mathrm{AD}$ subjects (Table 2). Age and pNfL were also significantly correlated among the $29 \mathrm{CN}$ subjects who were negative for elevated amyloid and hippocampal atrophy $(r=0.58 ; p=$ 0.0009). Among AD subjects, pNfL and age were significantly correlated; this remained significant after controlling for CDR-SB $(r=0.42, p<0.0001)$. Normalized hippocampal volume was correlated with age in both the $\mathrm{CN}$ and $\mathrm{AD}$ groups, but the dichotomous measure for hippocampal atrophy was associated with age only in the AD subjects. Amyloid measures 
Table 2

Relationship between age and biomarkers in Cognitively Normal and AD Subject

\begin{tabular}{|c|c|c|}
\hline Measure & $\begin{array}{l}\text { Pearson Correlation } \\
\text { Coefficient }\end{array}$ & $p$ \\
\hline \multicolumn{3}{|l|}{ Cognitively Normal Subjects } \\
\hline pNfL*(pg/ml) (all CN, $n=51)$ & 0.44 & $<0.0001$ \\
\hline Amyloid $(n=41)$ & -0.02 & NS \\
\hline \multicolumn{3}{|l|}{ Elevated $(N / Y)$} \\
\hline Centiloids & 0.22 & NS \\
\hline Hippocampal Atrophy $(n=45)$ & 0.24 & NS \\
\hline \multicolumn{3}{|l|}{ Present $(N / Y)$} \\
\hline$H V / I C V$ & -0.39 & 0.007 \\
\hline \multicolumn{3}{|l|}{ Alzheimer's Disease Subjects } \\
\hline $\mathrm{pNfL}^{*}(\mathrm{pg} / \mathrm{ml})($ all $\mathrm{AD}, n=156)$ & 0.40 & $<0.0001$ \\
\hline \multicolumn{3}{|l|}{ Amyloid Positivity ( $n=132)$} \\
\hline Elevated $(N / Y)$ & -0.08 & NS \\
\hline Centiloids & -0.03 & NS \\
\hline \multicolumn{3}{|l|}{ Hippocampal Atrophy $(n=151)$} \\
\hline $\operatorname{Present}(N / Y)$ & 0.18 & 0.02 \\
\hline $\mathrm{HV} / \mathrm{ICV}$ & -0.35 & $<0.0001$ \\
\hline
\end{tabular}

$\mathrm{CN}$, cognitively normal; $\mathrm{HV}$, hippocampal volume; ICV, intracranial volume * pNfL was log transformed for analyses in this table.

were not correlated with age in either group of subjects (Table 2).

$\mathrm{CN}$ women had a higher pNfL level than men after adjusting for age (pNfL: $15.0 \pm 13.0$ versus $11.1 \pm$ $4.2 ; \mathrm{F}(2,48)=4.7 ; p=0.03)$, but there were no sex differences for $\mathrm{pNfL}$ in any of the three largest diagnostic subgroups: AD, MD, or Dx-Other groups. There was no difference in pNfL between Hispanic and non-Hispanic subjects in either the $\mathrm{CN}, \mathrm{AD}$, neuropsychiatric, or Dx-Other diagnostic groups, adjusting for age.

\section{Association of pNfL with imaging biomarkers}

(Table 3)

In the entire cohort of subjects with cognitive impairment, pNfL was associated with binary and continuous measures of amyloid and hippocampal atrophy. In the subgroup with $\mathrm{AD}$, only normalized hippocampal volume was associated with $\mathrm{pNfL}$, while in the $\mathrm{CN}$ subjects, pNfL was not associated with any of the imaging measures.

\section{Biomarkers by etiological diagnosis (Table 4)}

$p N f L$

Among all subjects, after adjusting for age, pNfL was higher in the $\mathrm{AD}$ and FTLD groups compared to the $\mathrm{CN}$ group, using a post-hoc Dunnett Hsu comparison with $\mathrm{CN}$ as the control group (Table 4 and Fig. 1). An exploratory analysis revealed that subjects in the FTLD group had higher pNfL than those
Table 3

Relationship between pNfL and biomarkers for Atrophy and Amyloid Load

\begin{tabular}{lcc}
\hline Measure & $\begin{array}{c}\text { Pearson } \\
\text { Correlation } \\
\text { Coefficient }\end{array}$ & \\
& & \\
\hline All Subjects with Cognitive Impairment & & \\
Amyloid $(n=197)$ & & \\
$\quad$ Elevated $(N / Y)$ & 0.27 & $<0.0001$ \\
$\quad$ Centiloids & 0.18 & 0.01 \\
Hippocampal Atrophy $(n=245)$ & 0.32 & $<0.0001$ \\
$\quad$ Present $(N / Y)$ & & \\
HV/ICV & -0.35 & $<0.0001$ \\
Cognitively Normal Subjects & & \\
Amyloid $(n=41)$ & & \\
$\quad$ Elevated $(N / Y)$ & -0.19 & NS \\
$\quad$ Centiloids & 0.11 & NS \\
Hippocampal Atrophy $(n=45)$ & & \\
$\quad$ Present $(N / Y)$ & -0.10 & NS \\
HV/ICV & 0.02 & NS \\
Alzheimer's Disease Subjects & & \\
Amyloid Positivity $(n=132)$ & & \\
$\quad$ Elevated $(N / Y)$ & 0.20 & NS \\
Centiloids & 0.14 & NS \\
Hippocampal Atrophy $(n=151)$ & & \\
$\quad$ Present $(N / Y)$ & 0.04 & NS \\
HV/ICV & -0.36 & $<0.0001$ \\
\hline CN cogntively & &
\end{tabular}

$\mathrm{CN}$, cognitively normal; HV, hippocampal volume; ICV, intracranial volume * pNfL was log transformed for analyses in this table.

in the $\mathrm{AD}$ group after controlling for age $(F=9.4$, $\mathrm{df}=1 ; p=0.003$ ).

Using logistic regression, $\mathrm{pNfL}$ was a predictive marker for differentiating $\mathrm{CN}$ from $\mathrm{AD}$ subjects $\left(\chi^{2}=24.8, \mathrm{df}=1 ; p>0.0001\right)$ with an AUROC curve of 0.798 (95\% CI: 0.737-0.860). Using Youden's procedure and high pNfL levels as test positive for $\mathrm{AD}$, the optimal sensitivity/specificity was $76 \% / 73 \%$, demonstrating fair levels of discrimination. For the differentiation of $\mathrm{CN}$ from FTLD subjects, pNfL was predictive by logistic regression $\left(\chi^{2}=6.7, \mathrm{df}=1\right.$; $p=0.01)$ with an AUROC curve of 0.955 (95\% CI: $0.899-1.000)$. The optimal sensitivity/specificity was $100 \% / 84 \%$.

\section{Amyloid}

The frequency of positive amyloid PET was different between the groups $\left(\chi^{2}=67.5, \mathrm{df}=6 ; p<0.0001\right)$. Post-hoc comparisons with $\mathrm{CN}$ subjects $(14.6 \% \mathrm{~A}+)$ showed that there was a higher frequency of elevated amyloid only in the AD group (60.9\%). Using a continuous measure for amyloid load, i.e., centiloid units, a difference between groups was found to be present, after controlling for age, and a measure of functional severity, i.e., the CDR-SB $(\mathrm{F}(8,216)=8.76$, $p<0.0001$ ), with post-hoc Dunnett Hsu procedure 


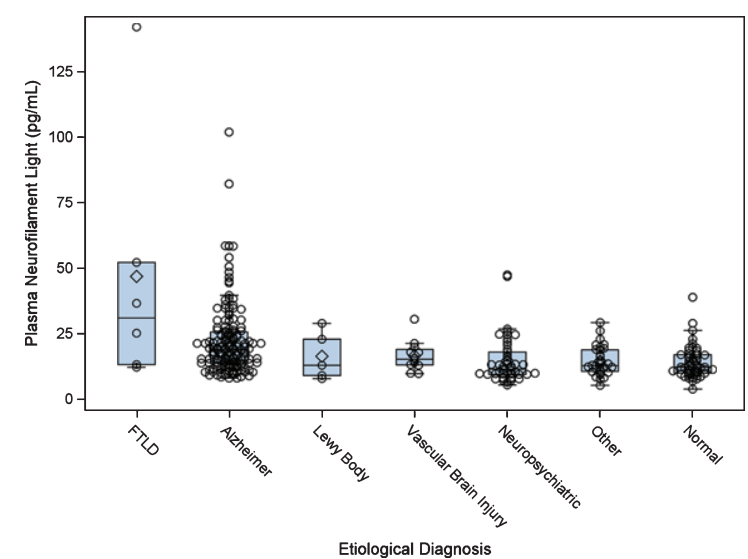

Fig. 1. Plasma neurofilament light by etiological diagnosis. Boxplots show the distribution of plasma neurofilament light by diagnostic category. FTLD, frontotemporal lobar degeneration.

showing that the AD group had higher centiloid values than the $\mathrm{CN}$ groups $(p=0.03)$ (Table 3$)$.

\section{Hippocampal atrophy}

The presence of hippocampal atrophy $\left(\chi^{2}=152.6\right.$, $\mathrm{df}=6 ; p<0.0001)$ was also different between the groups, with the post-hoc comparisons showing that the $\mathrm{CN}$ group had less atrophy than the AD group (90.1\% versus $\left.13.3 \%, \chi^{2}=102.2, \mathrm{df}=1 ; p<0.0001\right)$ and the FTLD group $\left(83.3 \%\right.$ versus $13.3 \%, \chi^{2}=12.4$, $\mathrm{df}=1 ; p<0.0001)$. Using continuous measures for hippocampal volume, there was a difference between groups after controlling for age $(\mathrm{F}(6,271)=10.3$, $p<0.0001)$. The $\operatorname{AD}(\mathrm{p} 0<.0001)$, FTLD $(p=0.03)$, LBD $(p=0.01)$, and Dx-Other $(p=0.002)$ groups had lower hippocampal volume compared to the $\mathrm{CN}$ group using a Dunnett $\mathrm{Hsu}$ procedure with the $\mathrm{CN}$ as the control group (Table 4).

\section{APOE}

The frequency of APOE4 positive subjects was different across diagnostic groups $\left(\chi^{2}=15.6, \mathrm{df}=6\right.$; $p=0.02$ ). Post-hoc comparisons showing that subjects in the $\mathrm{CN}$ group had a lower frequency of APOE4 positivity (13.3\%) than those in the AD group $(90.1 \%)$.

Table 5 shows the results of a GLM, with CDRSB score at baseline as the dependent variable, age as a covariate, and pNfL concentration, binary status for both elevated amyloid and hippocampal atrophy, and the number of APOE $\varepsilon 4$ alleles as independent predictor variables. In that model, all biomarker variables were significant predictors of severity of impairment, after controlling for the other variables. 
Table 5

Prediction of CDR sum of boxes at baseline, using pNfL, Imaging Biomarkers, and APOE genotype

\begin{tabular}{|c|c|c|c|c|c|c|c|c|c|}
\hline \multirow[t]{2}{*}{ Variable } & \multicolumn{3}{|c|}{$\begin{array}{l}\text { Models with } \\
\text { one biomarker }\end{array}$} & \multicolumn{3}{|c|}{$\begin{array}{c}\text { Model with } \\
\text { Imaging Biomarkers }\end{array}$} & \multicolumn{3}{|c|}{$\begin{array}{c}\text { Model with } \\
\text { all Biomarkers }\end{array}$} \\
\hline & $\mathrm{df}$ & $\begin{array}{l}\text { Type III } \\
\text { Mean } \\
\text { Square }\end{array}$ & $\mathrm{F}$ & df & $\begin{array}{l}\text { Type III } \\
\text { Mean } \\
\text { Square }\end{array}$ & $\mathrm{F}$ & $\mathrm{df}$ & $\begin{array}{l}\text { Type III } \\
\text { Mean } \\
\text { Square }\end{array}$ & $\mathrm{F}$ \\
\hline Amyloid Status (A + versus A-) & 1,225 & 152.8 & $32.0^{* * *}$ & 1,224 & 97.2 & $22.5^{* * *}$ & 1,223 & 74.8 & $18.7^{* * *}$ \\
\hline Hippocampal Atrophy (HP+versus Hp-) & 1,225 & 164.0 & $34.7^{* * *}$ & 1,224 & 108.4 & $25.2^{* * *}$ & 1,223 & 84.5 & $21.1^{* * *}$ \\
\hline Plasma NfL & 1,224 & 138.2 & $28.5^{* * *}$ & & & & 1,223 & 42.9 & $18.5^{* * *}$ \\
\hline Age & & & & 1,224 & 0.0 & 0.0 & 1,223 & 11.6 & 2.9 \\
\hline$A P O E$ (\# of $\varepsilon 4$ alleles) & & & & 1,224 & 7.1 & 1.6 & 1,223 & 9.1 & 2.3 \\
\hline Model $\mathrm{R}^{2}$ & \multicolumn{3}{|c|}{$\begin{array}{c}14.5 \text { (pNfL) } \\
16.5 \text { (Hippocampal } \\
\text { atrophy) } \\
5.6 \text { (Amyloid status) }\end{array}$} & 24.1 & & & 29.9 & & \\
\hline
\end{tabular}

All models include age and $A P O E$ as covariates. Only subjects will all imaging biomarkers are included in these models. ${ }^{*} p<0.05 ;{ }^{* *} p<0.01$; ${ }^{* *} p<0.001 ; A+$ versus A-: elevated versus not elevated amyloid; Hp + versus Hp-: positive versus negative for hippocampal atrophy.

Table 6

Prediction of change in CDR sum of boxes from baseline value to year 2, using pNfL and imaging biomarkers in cognitively impaired subjects

\begin{tabular}{|c|c|c|c|c|c|c|}
\hline \multirow[t]{2}{*}{ Variable } & \multicolumn{3}{|c|}{$\begin{array}{c}\text { Model with pNfL, imaging } \\
\text { biomarkers, memory score, age } \\
\text { and } A P O E \text { genotype }\end{array}$} & \multicolumn{3}{|c|}{$\begin{array}{c}\text { Model with pNfL, MRI imaging } \\
\text { biomarker, memory score, age } \\
\text { and } A P O E \text { genotype }\end{array}$} \\
\hline & df & $\begin{array}{c}\text { Type III } \\
\text { Mean Square }\end{array}$ & $\begin{array}{l}\text { F for interaction } \\
\text { with time }\end{array}$ & $\mathrm{df}$ & $\begin{array}{c}\text { Type III } \\
\text { Mean Square }\end{array}$ & $\begin{array}{l}\text { F for interaction } \\
\text { with time }\end{array}$ \\
\hline Amyloid Status $(A+$ versus A- $)$ & 2,178 & 16.4 & $7.6^{* *}$ & & & \\
\hline Hippocampal Atrophy (HP+versus Hp-) & 2,178 & 3.8 & 1.8 & 2,178 & 5.4 & 2.3 \\
\hline Plasma NfL & 2,178 & 4.4 & 2.0 & 2,178 & 8.7 & $3.8^{*}$ \\
\hline Age & 2,178 & 2.4 & 1.1 & 2,178 & 2.7 & 1.2 \\
\hline$A P O E$ (\# of $\varepsilon 4$ alleles) & 2,178 & 4.2 & 1.9 & 2,178 & 2.7 & 1.2 \\
\hline HVLT-R & 2,178 & 19.9 & $9.2^{* * *}$ & 2,178 & 32.4 & $14.0^{* * *}$ \\
\hline
\end{tabular}

${ }^{*} p<0.05 ;{ }^{* *} p<0.01 ;{ }^{* * *} p<0.001$. Only subjects with all imaging biomarkers are included in these models. $A+$ versus A-: elevated versus not elevated amyloid; Hp + versus Hp-: positive versus negative for hippocampal atrophy; HVLT-R, Hopkins Verbal Learning TestRevised.

The total variance explained was $29.9 \%$ with $\mathrm{pNfL}$ in the model. When pNfL was removed from the model, the variance explained was $24.1 \%$. When these analyses were conducted with continuous measures for amyloid load (centloid) and hippocampal atrophy (volume normalized to ICV), the model with imaging biomarkers had a $\mathrm{R}^{2}$ of $27.0 \%$; when $\mathrm{pNfL}$ was added to the model, $\mathrm{R}^{2}$ increased to 33.9 and $\mathrm{pNfL}$ was a significant predictor of CDR-SB after controlling for other variables $(F=22.2 ; p<0.0001)$.

\section{Prediction of cognitive/functional decline: Added value of $p N f L$}

Change in the CDR-SB scores over a mean followup period of $2.3 \pm 0.3$ years was correlated with the baseline value of all three biomarkers: amyloid status $(r=-0.53, p<0.0001)$, hippocampal atrophy status $(r=-0.43, p<0.0001)$ and $\log$ transformed
$\mathrm{pNfL}(\mathrm{r}=-0.44, p<0.0001)$, as well as with the score on the HVLT-R $(r=0.58, p<0.0001)$. In a repeated measures GLM among 96 cognitively impaired subjects who had at least 2 follow-up visits, score on HVLT-R and amyloid status, but not pNfL or hippocampal atrophy status, were predictors of decline on the CDR-SB from baseline to visit 2, independent of the other variables in the model, including age and APOE (Table 6). In the same group of subjects, when amyloid status was dropped from the model, HVLT-immediate and $\mathrm{pNfL}$ were predictive of decline, independent of the other variables. Figure 2 shows three ROC curves for the prediction of a decline of two or more points on the CDR-SB over 2 follow-up visits. In three separate models, using baseline measures of 1) pNfL, 2) HVLT-R, and 3) both pNfL and HVLT-R as predictors, the AUROC curves were 0.821 (95\% CI: 0.734-0.909), 0.825 (95\% CI: 0.741-0.910), and 0.866 (95\% CI: 


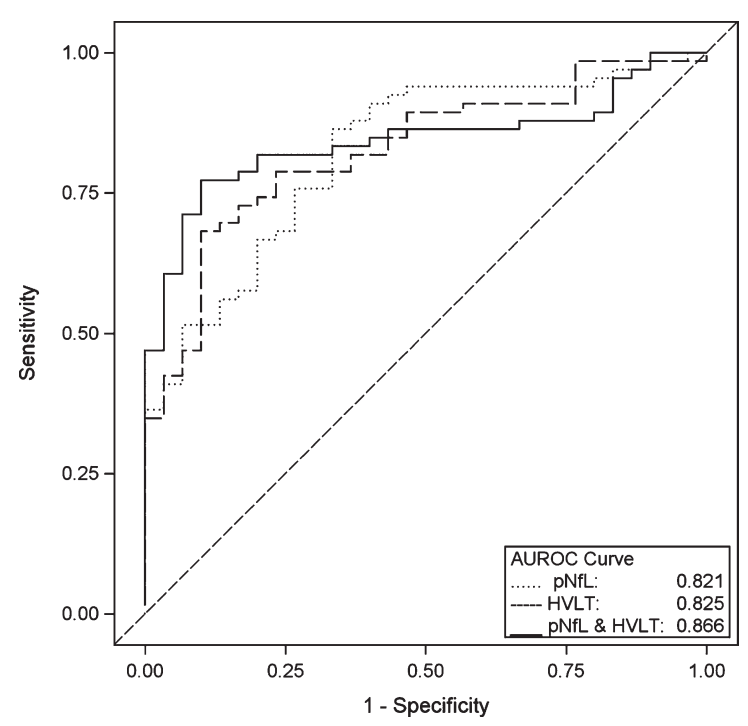

Fig. 2. ROC Curves for Prediction of Decline on the CDR. Area under the receiver operating characteristic (AUROC) curve for prediction of a decline of 2 or more points on the CDR sum of boxes in cognitively impaired subjects over 2 follow-up visits (an average of 2.3 years), using baseline measures of Hopkins Verbal Learning Test-Revised (HVLT-R) total recall, plasma neurofilament Light (pNfL) and the combination of both HVLT-R and pNfL. The logistic regression models were adjusted for age and $A P O E$ $\varepsilon 4$ genotype.

$0.792-0.940)$. The corresponding optimal sensitivities/specificities at a minimum $80 \%$ sensitivity were $80.3 \% / 66.6 \%, 80.3 \% / 63.3 \%$, and $80.3 \% / 80.0 \%$.

\section{DISCUSSION}

Within the ethnically diverse 1 Florida ADRC cohort, we investigated the relationship between $\mathrm{pNfL}$ and both demographic and clinical characteristics. Consistent with prior research, we found that $\mathrm{pNfL}$ increases with age, amyloid load, and hippocampal atrophy, and is elevated in those with neurodegenerative diseases including AD and FTLD (Tables 2-4). In our cohort, pNfL was not related to Hispanic ethnicity and was related to sex only in $\mathrm{CN}$ subjects, where women had higher levels. We also showed that $\mathrm{pNfL}$ may have a complementary and supportive role to brain imaging and cognitive testing in a memory disorder evaluation, although its diagnostic sensitivity and specificity appears to be modest as a stand-alone measure.

The association of pNfL with Hispanic ethnicity was explored because we have found differences between older Hispanic and non-Hispanic adults in the Miami area on other biological measures. For example, it was found that the association of $A P O E$ $\varepsilon 4$ allele to amyloid load was weaker in Hispanics as compared to non-Hispanic whites [19]. We also showed that the $A P O E$ dose effect on risk for $\mathrm{AD}$ may be greater in non-Hispanics compared to Hispanics [24]. In the current study, we found no effect of Hispanic ethnicity on pNfL in the entire 1Florida ADRC cohort or any diagnostic subgroup.

Increased age was associated with greater $\mathrm{pNfL}$ in cognitively normal subjects, a finding that remained even among those normal subjects without $\mathrm{AD}$ pathology on brain imaging. Among AD subjects, there was also an age-related increase in pNfL (Table 2). A recent meta-analysis showed that CSF NfL increased with age in CN subjects and in many neurological conditions, including AD [7]. The findings in this study also suggest that the association between age and biomarkers may be greater for NfL than for MRI and amyloid PET biomarkers. As a result of age-related increases in pNfL and $\mathrm{cNfL}$ across $\mathrm{CN}$ and $\mathrm{AD}$ subjects, reference ranges for NfL may have to be age-specific for clinical use. In addition, $\mathrm{CN}$ subjects with elevated NfL may score lower on cognitive tests, which could have an effect on the calculation of cutoffs for normative data for neuropsychological tests [6]. It has also been suggested that better normative data could be obtained by excluding "cognitively normal" subjects with elevated amyloid [25]. We found that $\mathrm{CN}$ women had higher pNfL than men, contrary to a recent metaanalysis which found higher CSF NfL for men than women in both $\mathrm{CN}$ and $\mathrm{AD}$ subjects [7]. The role of sex in NfL should be further studied.

Consistent with other studies, pNfL was associated with level of cognitive and functional impairment as measured with CDR-SB [5]. Additionally, in this study we showed that impairment was associated with pNfL after controlling for imaging biomarkers, which themselves are predictive measures of severity of impairment (Table 5). This implies that the pathology associated with $\mathrm{pNfL}$ is additive to that associated with neurodegeneration measured on MRI and amyloid measured on PET, in regards to its effect on global cognition and function. The combination of reduced $A \beta_{42}$ and elevated pNfL in plasma was shown to be a strong risk factor for subsequent $\mathrm{AD}$ and all-cause dementia [26] in a large epidemiological study of non-demented subjects. Using the ATN framework, subjects with combined biomarkers have been shown to be more cognitively impaired and at greater risk for cognitive decline, using imaging and blood-based biomarkers [27, 28]. 
However, there are no studies directly showing that subjects with elevated $\mathrm{pNfL}$ have additional risk for cognitive/functional impairment in the presence of both hippocampal atrophy and elevated amyloid. A neuropathology study showed that NfL may influence cognition through a non-AD pathway, namely, cerebral atherosclerosis [28]. An initial estimate of the utility of pNfL as a stand-alone measure was obtained by computing its ability to discriminate $\mathrm{CN}$ subjects from those subjects with a diagnosis of AD. The AUROC was 0.80 with a modest sensitivity/specificity of $75 \% / 73 \%$. This is consistent with an AUROC for ADNI of 0.79 for pNfL differentiating between $\mathrm{CN}$ and $\mathrm{AD}$ dementia [5].

Compared to cognitively normal control subjects, in this study, pNfL was elevated to the greatest degree in those with FTLD and to a lesser extent in those diagnosed with AD (Table 4), but not among those with cognitive impairment attributed to neuropsychiatric disorders, such as anxiety and depression, VBI, LBD, or a miscellaneous category, Dx-Other. Elevated NfL among subjects with FTLD and AD has been shown previously, as has increased NfL among subjects with many other neurological conditions, including vascular brain injury and LBD [7]. It should be noted however, that the sample sizes in this cohort may have been too small to find an effect for those subjects in this study.

Among subjects with cognitive impairment, baseline pNfL, elevated amyloid, hippocampal atrophy, and score on a memory test (HVLT-R) were correlated with cognitive and functional decline as measured by the CDR-SB over a 2.3-year period. However, in a model with all biomarkers and memory score as predictors, only amyloid status and memory score independently added to the prediction of decline. Since amyloid PET is rarely available outside of a research setting, another model without amyloid status was performed; in that model, both pNfL and memory score were independent predictors of decline, but hippocampal atrophy was not (Table 6). This underscores the well-known role of memory testing in clinical evaluation and the potential role of pNfL as an adjunct to imaging and to the ATN framework. In the ATN framework for a biological definition of $\mathrm{AD}$, the "N" stands for neurodegeneration or neuronal injury [29]. The " $\mathrm{N}$ " is usually measured with structural MRI, FDG PET, or total tau levels in CSF. These measures, like NfL, are not specific for $\mathrm{AD}$, but are used to stage disease severity and prediction of cognitive decline. Using those standards, the current study and prior investigations [3, 5] support a role for $\mathrm{NfL}$ in the ATN classification. Other pathways outside the ATN framework which influence cognitive decline include cerebrovascular disease [30], limbic-predominant age-related TDP-43 encephalopathy (LATE) [31], and primary age-related tauopathy (PART) [32]. In addition, factors, such as cognitive reserve [33], genetic influences, immune dysfunction, and individual lifestyles may either enhance or suppress the association of cognitive status to the underlying neuropathology [34].

In addition to demonstrating that $\mathrm{pNfL}$ may have a complementary role to neuroimaging, another strength of the current study was a relatively large group of subjects $(n=47)$ who were diagnosed with cognitive disorder with a primary neuropsychiatric etiology. Their mean pNfL level was not different from $\mathrm{CN}$ subjects, after controlling for age (Table 4). Further, their CDR-SB score at baseline and their change in CDR-SB at follow-up visits was not different from $\mathrm{CN}$ subjects, although they had lower scores on a list-learning test. In this group, the frequency of abnormal biomarkers is equivalent to that for $\mathrm{CN}$ subjects. In the absence of expensive neuroimaging tests, $\mathrm{pNfL}$ could be a valuable biomarker for differentiating those patients presenting with cognitive complaints who have neuropsychiatric symptoms versus those with neurodegenerative disease [35]. Similarly, pNfL may have diagnostic utility for subjects presenting with miscellaneous conditions such as sleep disorders and other non-neurodegenerative disorders, included in the Dx-Other group.

Limitations of the study include a small number of African American participants, the lack of serial pNfL measurements, the small number of participants with non-AD neurodegenerative disorders, and the relatively short longitudinal assessment interval. In addition, the Hispanics in this study were mostly from Cuba and South America and most self-reported as being white. Therefore, the results may not be generalizable to other Hispanic groups composed of people from Central America and other countries in the Caribbean.

Our findings suggest that $\mathrm{pNfL}$ provides a convenient marker of the severity of neurodegeneration and is associated with global cognitive and functional impairment, independent of hippocampal atrophy and abnormal amyloid load. An elevated pNfL concentration, similar to an elevated cholesterol, fasting glucose, or blood pressure measurement, could indicate the need for more detailed diagnostic evaluation and the institution of therapeutic and behavioral 
interventions. Plasma NFL was also shown to have utility in the prediction of cognitive and functional decline, beyond that provided by current standard of care assessments, such as brain imaging and cognitive testing. The clinical utility of pNfL as a marker of neurodegeneration is limited by its lack of specificity for AD since disorders such as FTLD also evidence elevated levels.

\section{ACKNOWLEDGMENTS}

This research was supported by the National Institute of Aging Grant numbers 5P50AG0477266021 Florida Alzheimer's Disease Research Center (Todd Golde, PI) and 1P30AG066506-01 Florida Alzheimer's Disease Research Center (Todd Golde, PI).

Authors' disclosures available online (https:// www.j-alz.com/manuscript-disclosures/20-0901r1).

\section{REFERENCES}

[1] Boyle PA, Yu L, Wilson RS, Leurgans E, Schneider JA, Bennett DA (2018) Person-specific contribution of neuropathologies to cognitive loss in old age. Ann Neurol 83,74-83.

[2] Sjogren M, Blomberg M, Jonsson M, Wahlund LO, Edman A, Lind K, Rosengren L, Blennow K, Wallin A (2001) Neurofilament protein in cerebrospinal fluid: A marker of white matter changes. J Neurosci Res 66, 510-516.

[3] Zetterberg H, Skillbäck T, Mattsson N, Trojanowski JQ, Portelius E, Shaw LM, Weiner MW, Blennow K; Alzheimer's Disease Neuroimaging Initiative (2016) Association of cerebrospinal fluid neurofilament light concentration with Alzheimer disease progression. JAMA Neurol 73, 60-67.

[4] Idland AV, Sala-Llonch R, Borza T, Watne LO, Wyller TB, Brækhus A, Zetterberg H, Blennow K, Walhovd KB, Fjell AM (2017) CSF neurofilament light levels predict hippocampal atrophy in cognitively healthy older adults. Neurobiol Aging 49, 138-144.

[5] Mattsson N, Andreasson U, Zetterberg H, Blennow K; Alzheimer's Disease Neuroimaging Initiative (2017) Association of plasma neurofilament light with neurodegeneration in patients with Alzheimer disease. JAMA Neurol 74, 557-566.

[6] Osborn KE, Khan OA, Kresge HA, Bown CW, Liu D, Moore EE, Gifford KA, Acosta LMY, Bell SP, Hohman TJ, Blennow K, Zetterberg H, Jefferson AL (2019) Cerebrospinal fluid and plasma neurofilament light relate to abnormal cognition. Alzheimers Dement (Amst), 11, 700-709.

[7] Bridel C, van Wieringen WN, Zetterberg H, Tijms BM, Teunissen CE, and the NFL Group (2019) Diagnostic value of cerebrospinal fluid neurofilament light protein in neurology: A systematic review and meta-analysis. JAMA Neurol 76, 1035-1048.

[8] Duara R, Loewenstein DA, Greig MT, Potter E, Barker W, Raj A, Schinka J, Borenstein A, Schoenberg M, Wu Y, Banko J, Potter H (2011) Pre-MCI and MCI: Neuropsy- chological, clinical, and imaging features and progression rates. Am J Geriatr Psychiatry 19, 951-960.

[9] Loewenstein DA, Greig MT, Schinka JA, Barker W, Shen Q, Potter E, Raj A, Brooks L, Varon D, Schoenberg M, Banko J, Potter H, Duara R (2012) An investigation of PreMCI: Subtypes and longitudinal outcomes. Alzheimers Dement $\mathbf{8}$, 172-179.

[10] Besser L, Kukull W, Knopman DS, Besser L, Kukull W, Knopman DS, Chui H, Galasko D, Weintraub S, Jicha G, Carlsson C, Burns J, Quinn J, Sweet RA, Rascovsky K, Teylan M, Beekly D, Thomas G, Bollenbeck M, Monsell S, Mock C, Zhou XH, Thomas N, Robichaud E, Dean M, Hubbard J, Jacka M, Schwabe-Fry K, Wu J, Phelps C, Morris JC (2018) Version 3 of the National Alzheimer's Coordinating Center's Uniform Data Set. Alzheimer Dis Assoc Disord 32, 351-358.

[11] Morris JC (1993) The Clinical Dementia Rating (CDR): Current version and scoring rules. Neurology 43, 24122412.

[12] O'Bryant SE, Lacritz LH, Hall J, Waring SC, Chan W, Khodr ZG, Massman PJ, Hobson V, Cullum CM (2010) Validation of the new interpretive guidelines for the clinical dementia rating scale sum of boxes score in the national Alzheimer's coordinating center database. Arch Neurol 67, 746-749.

[13] National Research Council (2004) Measuring Racial Discrimination. The National Academies Press, Washington, DC.

[14] Benedict RH, Schretlen D, Groninger L, Brandt J (1998) Hopkins verbal learning test-revised: Normative data and analysis of inter-form and test-retest reliability. Clin $\mathrm{Neu}$ ropsychol 12, 43-55.

[15] Folstein MF, Folstein SE, McHugh PR (1975) Mini-mental state: A practical method for grading the cognitive state of patients for the clinician. J Psychiatr Res 12, 189-198.

[16] McKhann GM, Knopman DS, Chertkow H, McKhann GM, Knopman DS, Chertkow H, Hyman BT, Jack CR Jr, Kawas $\mathrm{CH}$, Klunk WE, Koroshetz WJ, Manly JJ, Mayeux R, Mohs RC, Morris JC, Rossor MN, Scheltens P, Carrillo MC, Thies B, Weintraub S, Phelps CH (2011) The diagnosis of dementia due to Alzheimer's disease: Recommendations from the National Institute on Aging-Alzheimer's Association workgroups on diagnostic guidelines for Alzheimer's disease. Alzheimers Dement 7, 263-269.

[17] Duara R, Loewenstein DA, Potter E, Appel J, Greig MT, Urs R, Shen Q, Raj A, Small B, Barker W, Schofield E, Wu Y, Potter H (2008) Medial temporal lobe atrophy on MRI scans and the diagnosis of Alzheimer disease. Neurology 71, 1986-1992.

[18] Desikan RS, Cabral HJ, Hess CP, Dillon WP, Glastonbury CM, Weiner MW, Schmansky NJ, Greve DN, Salat DH, Buckner RL, Fischl B; Alzheimer's Disease Neuroimaging Initiative (2009) Automated MRI measures identify individuals with mild cognitive impairment and Alzheimer's disease. Brain 132, 2048-2057.

[19] Duara R, Loewenstein DA, Lizarraga G, Adjouadi M, Barker WW, Greig-Custo MT, Rosselli M, Penate A, Shea YF, Behar R, Ollarves A, Robayo C, Hanson K, Marsiske M, Burke S, Ertekin-Taner N, Vaillancourt D, De Santi S, Golde T, DeKosky S (2019) Effect of age, ethnicity, sex, cognitive status and APOE genotype on amyloid load and the threshold for amyloid positivity. Neuroimage Clin 22, 101800 .

[20] Sabri O, Seibyl J, Rowe C, Barthel H (2015) Beta-amyloid imaging with florbetaben. Clin Transl Imaging 3, 13-26. 
[21] Rowe CC, Doré V, Jones G, Baxendale D, Mulligan RS, Bullich S, Stephens AW, De Santi S, Masters CL, Dinkelborg L, Villemagne VL (2017) ${ }^{18}$ F-Florbetaben PET beta-amyloid binding expressed in Centiloids. Eur J Nucl Med Mol Imaging 44, 2053-2059.

[22] Navitsky M, Joshi AD, Kennedy I, Klunk WE, Rowe CC, Wong DF, Pontecorvo MJ, Mintun MA, Devous MD Sr (2018) Standardization of amyloid quantitation with florbetapir standardized uptake value ratios to the Centiloid scale. Alzheimers Dement 14, 1565-1571.

[23] Samtani MN, Raghavan N, Novak G, Nandy P, Narayan VA (2014) Disease progression model for Clinical Dementia Rating-Sum of Boxes in mild cognitive impairment and Alzheimer's subjects from the Alzheimer's Disease Neuroimaging Initiative. Neuropsychiatr Dis Treat 10, 929-52.

[24] Harwood DG, Barker WW, Ownby RL, St. George-Hyslop P, Duara R (2004) Apolipoprotein E polymorphism and age of onset for Alzheimer's disease in a bi-ethnic sample. Int Psychogeriatr 16, 317-326.

[25] Bos I, Vos SJB, Jansen WJ, Vandenberghe R, Gabel S, Estanga A, Ecay-Torres M, Tomassen J, den Braber A, Lleó A, Sala I, Wallin A, Kettunen P, Molinuevo JL, Rami L, Chetelat G, de la Sayette V, Tsolaki M, Freund-Levi Y, Johannsen P; Alzheimer's Disease Neuroimaging Initiative, Novak GP, Ramakers I, Verhey FR, Visser PJ (2018) Amyloid- $\beta$, tau, and cognition in cognitively normal older individuals: Examining the necessity to adjust for biomarker status in normative data. Front Aging Neurosci 10, 193.

[26] de Wolf F, Ghanbari M, Licher S, McRae-McKee K, Gras L, Weverling GJ, Wermeling P, Sedaghat S, Ikram MK, Waziry R, Koudstaal W, Klap J, Kostense S, Hofman A, Anderson R, Goudsmit J, Ikram MA (2020) Plasma tau, neurofilament light chain and amyloid- $\beta$ levels and risk of dementia; a population-based cohort study. Brain 143, 1220-1232.

[27] Altomare D, de Wilde A, Ossenkoppele R, Pelkmans W, Bouwman F, Groot C, van Maurik I, Zwan M, Yaqub M, Barkhof F, van Berckel BN, Teunissen CE, Frisoni GB, Scheltens P, van der Flier WM (2019) Applying the ATN scheme in a memory clinic population: The ABIDE project. Neurology 93, e1635-e1646

[28] Jack CR Jr, Wiste HJ, Therneau TM, Weigand SD, Knopman DS, Mielke MM, Lowe VJ, Vemuri P, Machulda MM, Schwarz CG, Gunter JL, Senjem ML, Graff-Radford J, Jones DT, Roberts RO, Rocca WA, Petersen RC (2019) Associations of amyloid, tau, and neurodegeneration biomarker profiles with rates of memory decline among individuals without dementia. JAMA 321, 2316-2325.
[29] Jack CR Jr, Bennett DA, Blennow K, Carrillo MC, Dunn B, Haeberlein SB, Holtzman DM, Jagust W, Jessen F, Karlawish J, Liu E, Molinuevo JL, Montine T, Phelps C, Rankin KP, Rowe CC, Scheltens P, Siemers E, Snyder HM, Sperling R; Contributors (2018) NIA-AA Research Framework: Toward a biological definition of Alzheimer's disease. Alzheimers Dement 14, 535-562.

[30] Luchsinger JA, Reitz C, Honig LS, Tang MX, Shea S, Mayeux R (2005) Aggregation of vascular risk factors and risk of incident Alzheimer disease. Neurology 65, 545-551.

[31] Nelson PT, Dickson DW, Trojanowski JQ, Jack CR, Boyle PA, Arfanakis K, Rademakers R, Alafuzoff I, Attems J, Brayne C, Coyle-Gilchrist IT, Chui HC, Fardo DW, Flanagan ME, Halliday G, Hokkanen SR, Hunter S, Jicha GA, Katsumata Y, Kawas CH, Keene CD, Kovacs GG, Kukull WA, Levey AI, Makkinejad N, Montine TJ, Murayama S, Murray ME, Nag S, Rissman RA, Seeley WW, Sperling RA, White Iii CL, Yu L, Schneider JA (2019) Limbicpredominant age-related TDP-43 encephalopathy (LATE): Consensus working group report. Brain 142, 1503-1527.

[32] Crary JF, Trojanowski JQ, Schneider JA, Abisambra JF, Abner EL, Alafuzoff I, Arnold SE, Attems J, Beach TG, Bigio EH, Cairns NJ, Dickson DW, Gearing M, Grinberg LT, Hof PR, Hyman BT, Jellinger K, Jicha GA, Kovacs GG, Knopman DS, Kofler J, Kukull WA, Mackenzie IR, Masliah E, McKee A, Montine TJ, Murray ME, Neltner JH, SantaMaria I, Seeley WW, Serrano-Pozo A, Shelanski ML, Stein T, Takao M, Thal DR, Toledo JB, Troncoso JC, Vonsattel JP, White CL 3rd, Wisniewski T, Woltjer RL, Yamada M, Nelson PT (2014) Primary age-related tauopathy (PART): A common pathology associated with human aging. Acta Neuropathol 128, 755-766.

[33] Stern, Y (2012) Cognitive reserve in ageing and Alzheimer's disease. Lancet Neurol 11, 1006-1012.

[34] Newcombe EA, Camats-Perna J, Silva ML, Valmas N, Huat TJ, Medeiros R (2018) Inflammation: The link between comorbidities, genetics, and Alzheimer's disease. J Neuroinflammation $\mathbf{1 5}, 276$.

[35] Katisko K, Cajanus A, Jääskeläinen O, Kontkanen A, Hartikainen P, Korhonen VE, Helisalmi S, Haapasalo A, Koivumaa-Honkanen H, Herukka SK, Remes AM, Solje E (2020) Serum neurofilament light chain is a discriminative biomarker between frontotemporal lobar degeneration and primary psychiatric disorders. J Neurol 267, 162-167. 